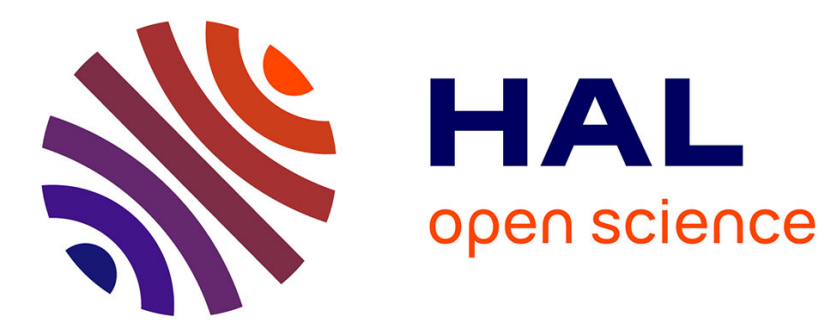

\title{
Rain dances, microstructure and transformations P. Clapp
}

\section{To cite this version:}

P. Clapp. Rain dances, microstructure and transformations. Journal de Physique IV Proceedings, 1994, 04 (C3), pp.C3-3-C3-11. 10.1051/.jp4:1994301 . jpa-00252498

\section{HAL Id: jpa-00252498 https://hal.science/jpa-00252498}

Submitted on 1 Jan 1994

HAL is a multi-disciplinary open access archive for the deposit and dissemination of scientific research documents, whether they are published or not. The documents may come from teaching and research institutions in France or abroad, or from public or private research centers.
L'archive ouverte pluridisciplinaire HAL, est destinée au dépôt et à la diffusion de documents scientifiques de niveau recherche, publiés ou non, émanant des établissements d'enseignement et de recherche français ou étrangers, des laboratoires publics ou privés. 


\title{
Rain dances, microstructure and transformations
}

\author{
P.C. CLAPP ${ }^{* * *}$ \\ ${ }^{*}$ CEREM, Saclay, France \\ ${ }^{* *}$ Center for Materials Simulation, Institute of Materials Science, 97 N. Eagleville Rd., Univ. of \\ Connecticut, Storrs, CT06269-3136, U.S.A.
}

\begin{abstract}
Ancient people employed witch doctors to perform ritual dances in the hope of inducing favorable phase transformations (rain) when and where they needed them. Cloud seeding with nucleation agents is now considered a slightly superior approach. However, we are still very far away from knowing how to "seed" a material to obtain the optimum transformation characteristics and consequent microstructure in many practically important applications. This is partly due to the fact that we do not yet understand how to predict or control the distribution of defects that will be the main agents of nucleation in a transforming material, assuming it is even known which defects are the critical ones in a given case! A survey of possible approaches to becoming a successful modern witch doctor will be presented.
\end{abstract}

\section{Introduction}

The understanding and control of phase transformations is often crucial to controlling material properties. This is true because not only does a phase transformation often radically alter the properties of a material (e. g. water to ice), but the resulting microstructure in addition may have important consequences for the material properties. It should be noted that phase transformations almost always need "seeds" (heterogeneous nuclei) to begin.

The distribution of "seeds" (nuclei) will strongly affect the transformation microstructure that results, and therefore the material properties. American Indian Rain Dances were attempts to induce a phase transformation (rain) in a particular location at a particular time. We now know that clouds can be "seeded" to produce rain almost on demand, if the clouds are available!

Some examples of phase transformations are:

1. Water to Ice (obvious, but still dramatic - i.e. it makes it possible to "walk on water")

2. Martensite in steel (causes tremendous strength and can help with toughness too)

3. Superconductivity (allows perpetual "frictionless" motion)

4. "Transformation toughening" in Ceramics (produces 10 to 20 -fold increase in toughness, e.g $\mathrm{ZrO} 2$, $\mathrm{Al} 2 \mathrm{O} 3+\mathrm{ZrO} 2)$.

In this paper we will discuss (with some brevity) all of the above transformations as illustrative examples of their type. Although it is an advantage that transformations can be controlled through the placement of nuclei, the disadvantage is that reproducibility can be difficult as anyone who has worked with shape memory alloys knows, where the memory effects depend very sensitively on the precise placement of nuclei. 
There are some important exceptions to this basic principle of being able to control phase transformations through the control of the distribution of nuclei. The first is if it is a higher order transformation (in the Ehrenfest classification), in which case the transformation has no latent heat, density discontinuity or hysteresis. Examples of this type are any of the transitions to the superconducting state and many of the ferroelectrics. In these cases, the transformation will inevitably begin when the transition temperature is reached and no nucleation process is required.

The second type of exception is in the very rare case of a first order transformation when, as a result of very careful elimination of impurities and other types of defects, homogeneous nucleation occurs. This usually requires a very deep and rapid quench as well. In this case then, all sites of the crystal are potential and active nucleation sites for the transformation. One classical example where this is believed to occur is in the solid state precipitation of coherent spherical single crystal particles of $\mathrm{Fe}$ (or $\mathrm{Co}$ ) from a $\mathrm{Cu}+2 \% \mathrm{Fe}$ (or $\mathrm{Co}$ ) homogenized alloy'.

\section{Il. Classical Nucleation Theory and the Cahn Diagram}

To understand how one might control the precipitate distribution through the grain size and surface chemistry of an alloy, a short review of nucleation theory is provided here which will lead to a useful diagram for controlling the spatial location of precipitates formulated by $\mathrm{Cahn}^{2}$. The approach followed here is taken from the excellent reference text by Christian ${ }^{3}$.

\section{II.1 Homogeneous Nucleation}

If one imagines an approximately spherical particle of the $\beta$ phase of radius $\mathbf{r}$, volume $\mathbf{V}_{\boldsymbol{\beta}}$, and surface area $A_{\beta}$ with a driving force of $\left(\mathbf{F}_{\beta}-\mathbf{F}_{\alpha}\right)$ attempting to grow at an arbitrary location in the interior of an $\alpha$ phase matrix, the free energy change of the total system (excluding strain energy contributions) will be :

$$
\text { where } \quad \begin{aligned}
& \Delta F=\left(F_{\beta}-F_{\alpha}\right) V_{\beta}+\sigma_{\alpha \beta} A_{\beta} \\
& V_{\beta}=f_{\beta} r^{3} ; f_{\beta} \cong 4 \pi / 3 \\
& A_{\beta}=f_{\alpha \beta} r^{2} ; f_{\alpha \beta} \cong 4 \pi
\end{aligned}
$$

and the $\mathbf{f}$ coefficients are known as "shape factors".

Since the positive surface energy term increases as $\mathbf{r}^{\mathbf{2}}$ and the negative volume term as $\mathbf{r}^{3}$, the total free energy of the system will always have to increase with increasing $\mathbf{r}$ before it can decrease, leading to a "nucleation barrier". By differentiating Eq. 1 with respect to $\mathbf{r}$ and setting it equal to zero, the critical barrier height $\Delta \mathbf{F}^{*}$ and critical nucleus radius $\mathbf{r}^{*}$ may be determined. They are:

$$
\Delta F^{*}=\frac{20 \sigma_{\alpha \beta}^{3} f_{\alpha \beta}^{3}}{27\left(F_{\alpha}-F_{\beta}\right)^{2} f_{\beta}^{2}}
$$

and

$$
r^{*}=\frac{2 \sigma_{\alpha \beta} f_{\alpha \beta}}{3\left(F_{\alpha}-F_{\beta}\right) f_{\beta}}
$$


One should notice that the nucleation barrier increases as the cube of the interfacial energy, and decreases only inversely as the square of the driving force.

The nucleation rate (in terms of critical nuclei per unit volume per second, $\mathbf{I}_{\mathbf{V}}$ ) is given by:

$$
\mathbf{I}_{\mathbf{v}}=\mathbf{N}_{\mathbf{v}} v \exp \left(-\Delta \mathbf{F}^{*} / \mathbf{k T}\right)
$$

where $\mathbf{N}_{\mathbf{v}}$ is the number of potential homogeneous nucleation sites (i.e. every atom) in the crystal; a number of the order of $10^{23} / \mathrm{cm}^{3}$. $v$ is the frequency at which atoms attempt to jump across the nuclear interface, and is generally taken to be of the order of the atomic vibration frequency, $10^{13} / \mathrm{sec}$. $\mathrm{k}$ is Boltzmann's constant and $\mathrm{T}$ is the absolute temperature. It is generally found, however, that most first order phase transformations (i.e. those with a latent heat, density discontinuity and hysteresis) do not nucleate homogeneously but heterogeneously, because the latter is often assisted by a substantially lower interfacial energy, which given Eqs. (3) and (4), produce a very large advantage in the nucleation rate for the heterogeneous mode, as will now be seen.

\section{II.2 Heterogeneous Nucleation}

Consider the configuration shown in Fig. 1 of a $\beta$ phase particle growing on a substrate $S$, but also partially imbedded in the $\alpha$ phase. Imagine that the interface of the $\beta$ particle with the $\alpha$ phase is a section of a spherical surface of radius $r$, and that the interface with the substrate is a circle. Let the "wetting" angle (i. e. the included angle between a tangent to the spherical surface at the contact point with the substrate, and the substrate itself) be $\boldsymbol{\theta}$. Furthermore it is assumed that the interfacial energies between the three solids are $\sigma_{\alpha \beta}, \sigma_{\alpha s}$ and $\sigma_{\beta s}$.

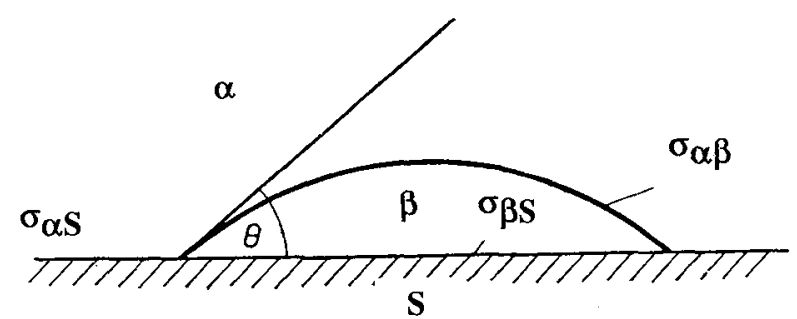

Fig. 1 - Nucleation of a $\beta$ particle in the $\alpha$ phase growing on a substrate $S$ (after Christian ${ }^{3}$ ).

The balance between surface tensions at the three phase junction yields:

$$
\sigma_{\alpha S}=\sigma_{\mathrm{gs}}+\sigma_{\alpha \beta} \cos \Theta
$$

Using this relation to eliminate two of the interfacial energies, it may be shown that the critical nucleation barrier for this type of heterogeneous nucleation is :

$$
\Delta F_{s}^{*}=\frac{4 \pi}{3} \frac{\sigma_{\alpha \beta}^{3}}{\left(F_{\alpha}-F_{\beta}\right)^{2}}\left(2-3 \cos \Theta+\cos ^{3} \Theta\right)
$$

and furthermore, for this case of spherical surfaces, taking Eq. (2) into account, one has:

$$
\Delta F_{S}^{*} / \Delta F^{*}=\left(2-3 \cos \Theta+\cos ^{3} \theta\right) / 4
$$


It is worth noting that as long as the wetting angle is less than $180^{\circ}$, the ratio in Eq. (7) is less than unity, showing that with any reasonable degree of wetting the nucleation barrier for this type of heterogeneous nucleation will be less than that for homogeneous nucleation.

Next we may consider the case of a $\beta$ particle nucleating at a grain boundary of the $\alpha$ phase, as shown in Fig. 2. In this situation there are only two relevant surface energies, $\sigma_{\alpha \alpha}$ and $\sigma_{\alpha \beta}$, and the wetting angle may be defined in an analogous fashion to the previous case. It may be shown ${ }^{3}$ that in this case the critical nucleation barrier $\Delta \mathbf{F}^{*} \mathbf{B}$ relative to the homogeneous barrier is given by:

$$
\Delta F_{B}^{*} / \Delta F^{*}=\left(2-3 \cos \theta+\cos ^{3} \theta\right) / 2
$$

and the surface tension balance gives the relation:

$$
\sigma_{\alpha \alpha}=2 \sigma_{\alpha \beta} \cos \Theta
$$

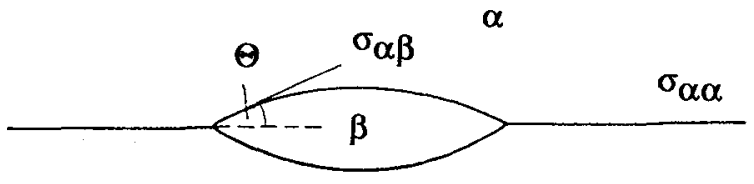

$\alpha$

Fig. 2 - Nucleation of a $\beta$ particle at an $\alpha$ grain boundary (after Clemm and Fisher ${ }^{4}$ )

Continuing on to cases where the $\beta$ particle nucleates at 3-grain or 4-grain junctions of the $\alpha$ phase, as illustrated in Fig. 3, more complicated but tractable expressions may be determined for the relative nucleation barriers ${ }^{3}$.

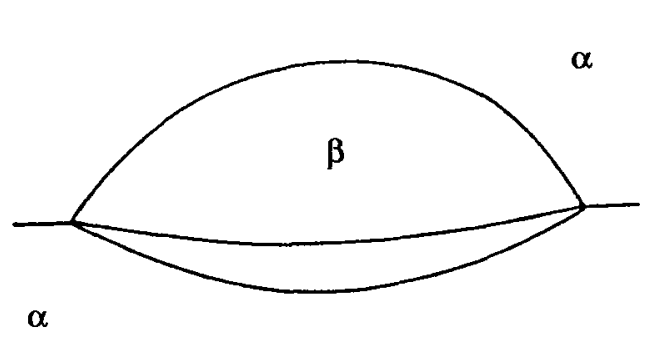

(a)

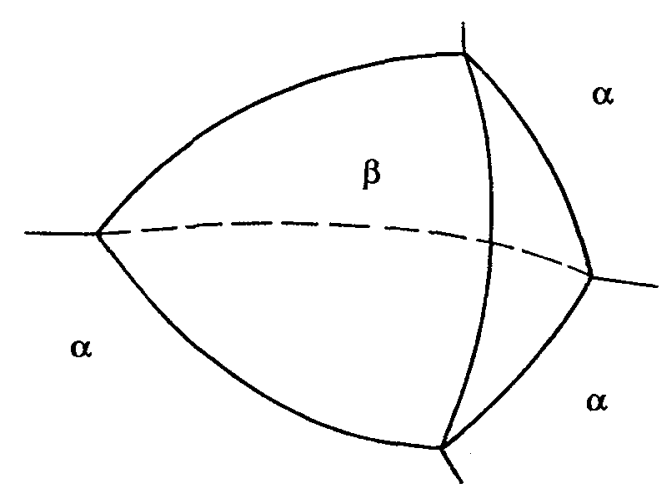

(b)

Fig. 3 - Shape of $\beta$ particle nucleating at (a) 3-grain junction and (b) 4-grain junction (after Clemm and Fisher ${ }^{4}$ )

All of this may be plotted, as is shown in Fig. 4, where it is clear that for any alloy chemistry (i.e. any chosen value of $\left.\cos \Theta=\sigma_{\alpha \alpha} / 2 \sigma_{\alpha \beta}\right)$, the 4-grain nucleus is lower in energy than the 3-grain, etc.

However the 4-grain junction has the disadvantage of being a much rarer site than the 3-grain or the 2grain, and their relative populations will turn out to be important (through the factor Ny) in determining the relative nucleation rates. These relative nucleation rates may be estimated by realizing that the relative number of potential nucleation sites at a 2-grain junction to that of all sites in the crystal is approximately given by the ratio of the grain boundary thickness $\delta$ to the grain diameter D. 
Thus in Eq. 4 for the nucleation rate for boundary particles the number of potential starting sites, $\mathbf{N V}$ is now to be multiplied by the factor $(\delta / \mathrm{D})$, for 3-grain junctions by $(\delta / \mathrm{D})^{2}$, and for 4 -grain by $(\delta / \mathrm{D})^{3}$. From high resolution microscopy and atom probe evidence $\delta$ is generally found to be two or three atomic diameters, or about $1 \mathrm{~nm}$ in a broad range of alloys. The value of $\mathrm{D}$, of course, is under the control of the experimenter to a reasonable degree, and will be an important control parameter in determining the distribution of nuclei. A quantity $\mathbf{R}^{\mathbf{B}}$ is defined as $\mathrm{kT}$ in $(\mathrm{D} / \delta)$. Using this kind of analysis, $\mathrm{Cahn}^{2}$ calculated the relative nucleation rates at each type of heterogeneous site to determine which would be the dominant form, the final result being shown in Fig. 5, the "Cahn diagram".

The way such a diagram is used is to realize that the alloy chemistry sets a value for $\sigma_{\alpha \alpha} / \sigma_{\alpha \beta}$, which needs to be roughly estimated in some way. This then fixes a value on the abscissa. As the alloy is cooled down below the first temperature at which precipitation can occur, the value of $\Delta \mathbf{F}^{*}$ steadily decreases from a very high value, meaning that the point representing the alloy rises along a vertical line in the diagram, starting from $\mathbf{R}^{\mathbf{B}} / \Delta \mathbf{F}^{*}=0$. Until it reaches the boundary line abcde that cuts diagonally across the diagram, the calculated nucleation rate will be less than 1 per $\mathrm{cm}^{3} / \mathrm{sec}$, which is considered to be practically unobservable. Thus, depending on the alloy chemistry (i.e. the position on the abscissa), corner, edge, boundary or homogeneous nucleation may be the first to be observed on slow cooling. This situation may be altered by quenching rapidly into any of the desired regions of the diagram passed through by the fixed vertical line. The grain size $D$, through its control of the parameter $\mathbf{R}^{\mathbf{B}}$, will also affect the outcome. Finally, it may be seen that although a region of observable homogeneous nucleation does show up on the Cahn diagram, it is nevertheless confined to a very narrow range of alloy chemistries and requires a very deep quench to reach.

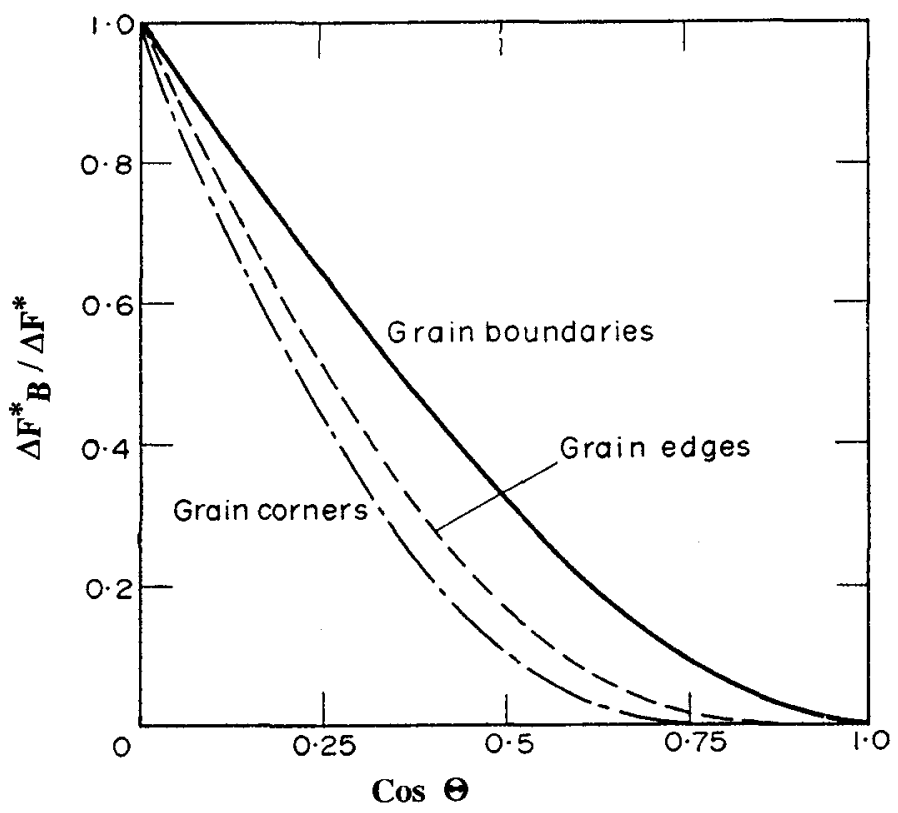

Fig. 4 - Ratio of the critical free energy barrier for nucleation at different numbers of grain junctions to that for homogeneous nucleation in the grain interior $\left(\mathrm{after} \mathrm{Cahn}^{2}\right)$. 


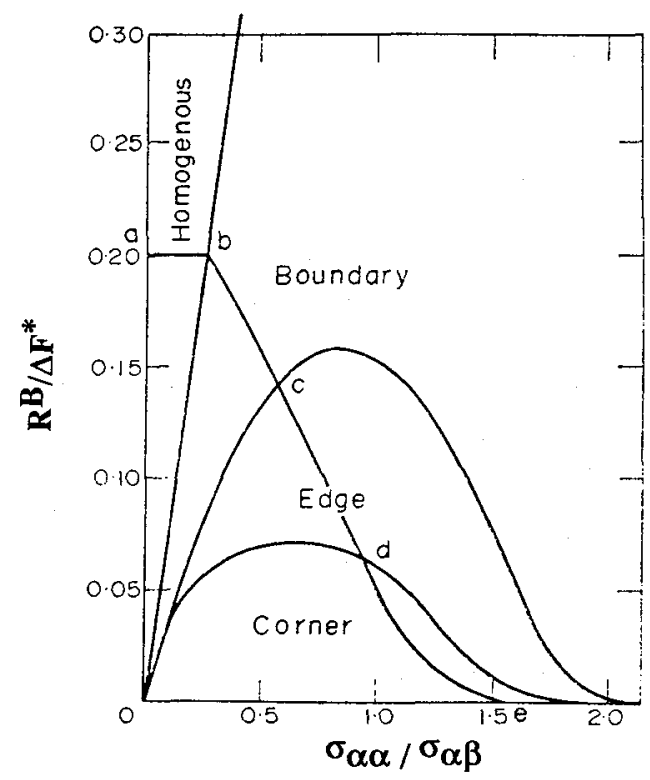

Fig. 5 - Plot of the conditions which would favor the dominance of different types of nucleation sites. Only for conditions above the curve abcde are nucleation rates considered to be experimentally observable (after $\left.\mathrm{Cahn}^{2}\right)$.

\section{Nucleation of Martensitic Transformations}

This is a type of transformation which can occur very rapidly, often at speeds approaching the speed of sound. Sometimes they are called "military transformations" because the atoms apparently move from one crystalline arrangement to the new configuration in the same coordinated manner as soldiers on a parade ground. Perhaps the best known example is that of the fcc to bct transformation in ferrous alloys, although the martensitic transformation which is responsible for the memory effect in shape memory alloys is becoming increasingly familiar. There is still a considerable debate regarding the precise manner in which a martensite phase nucleates, but there is accumulating evidence that it is basically a non-classical, usually heterogeneous process involving pre-existing strain centers and localized vibrational modes 5 . Although it is not yet known precisely which kinds of defects are the most effective for initiating a given martensitic transformation, the distribution of nucleation sites can be controlled in an empirical manner by thermomechanical treatments, such as those that have been used for centuries in modifying the properties of steels.

Apart from the strengthening effect which the strain field of martensite plates provide to the parent matrix (as in steels), a second beneficial property called "transformation toughening" can sometimes be obtained if the stress field of a propagating crack in the material can induce further transformation in its vicinity. This requires that the transformation occur with a small increase in volume (on the order of a few percent) and that the transformation be induced above or below the crack plane in order to press the crack closed, rather than directly ahead of the crack which would tend to pull the crack open. This phenomenon is very important in "TRIP" steels and the new high toughness ceramics (e. g. $\mathrm{ZrO}_{2}, \mathrm{Al}_{2} \mathrm{O}_{3}+\mathrm{ZrO}_{2}$, etc.). Composites can be made in which a fine distribution of particles of a transforming material (e. g. $\mathrm{ZrO}_{2}$ ) are embedded in a host material (e. g. $\mathrm{Al}_{2} \mathrm{O}_{3}$ ) thereby providing a toughening effect to the entire composite. In the process of designing new materials to take advantage of this phenomenon it is very important to know whether the martensite phase will be stress induced on either side of the crack plane (leading to an increase in toughness) or directly ahead of the crack (causing easier crack propagation). It has generally been supposed $^{6}$ that transformation will usually be stress induced in a "kidney shaped" zone as illustrated in Fig. 6. 


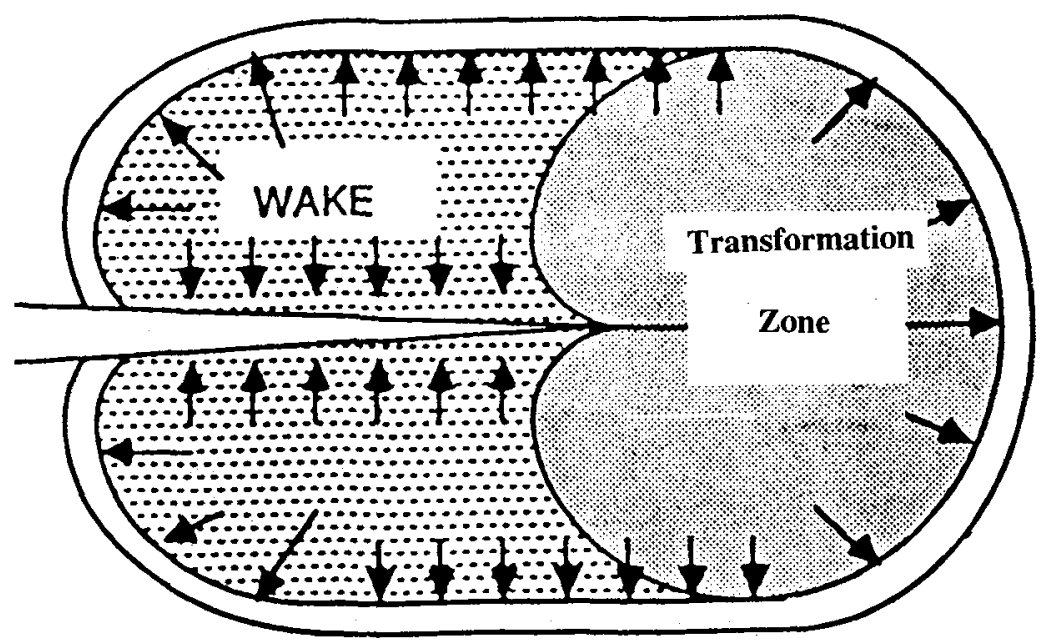

Fig. 6 - Zone of transformation expected under Mode I loading (stress normal to crack plane) assuming transformation is triggered wherever the principal stress is greater than some critical amount ( after Green et al. ${ }^{6}$ ).

However, by using a combined molecular dynamics-Monte-Carlo simulation method to study the details of the pattern of stress induced martensite in a NiAl ordered intermetallic alloy under Mode I loading, a rather different transformation distribution is found ${ }^{7,8}$ as shown in Fig. 7.

This will cause the estimated amount of transformation toughening to be substantially different than that calculated with a principal stress criterion. The implications of this difference are just now being worked out ${ }^{8}$, and the advantage of this modelling approach is that different parameters of the transformation (e. g. volume dilatation, principal shear, etc.) can be varied systematically to determine the resulting change in toughness, which ultimately may serve as a useful guide to the materials developer. 


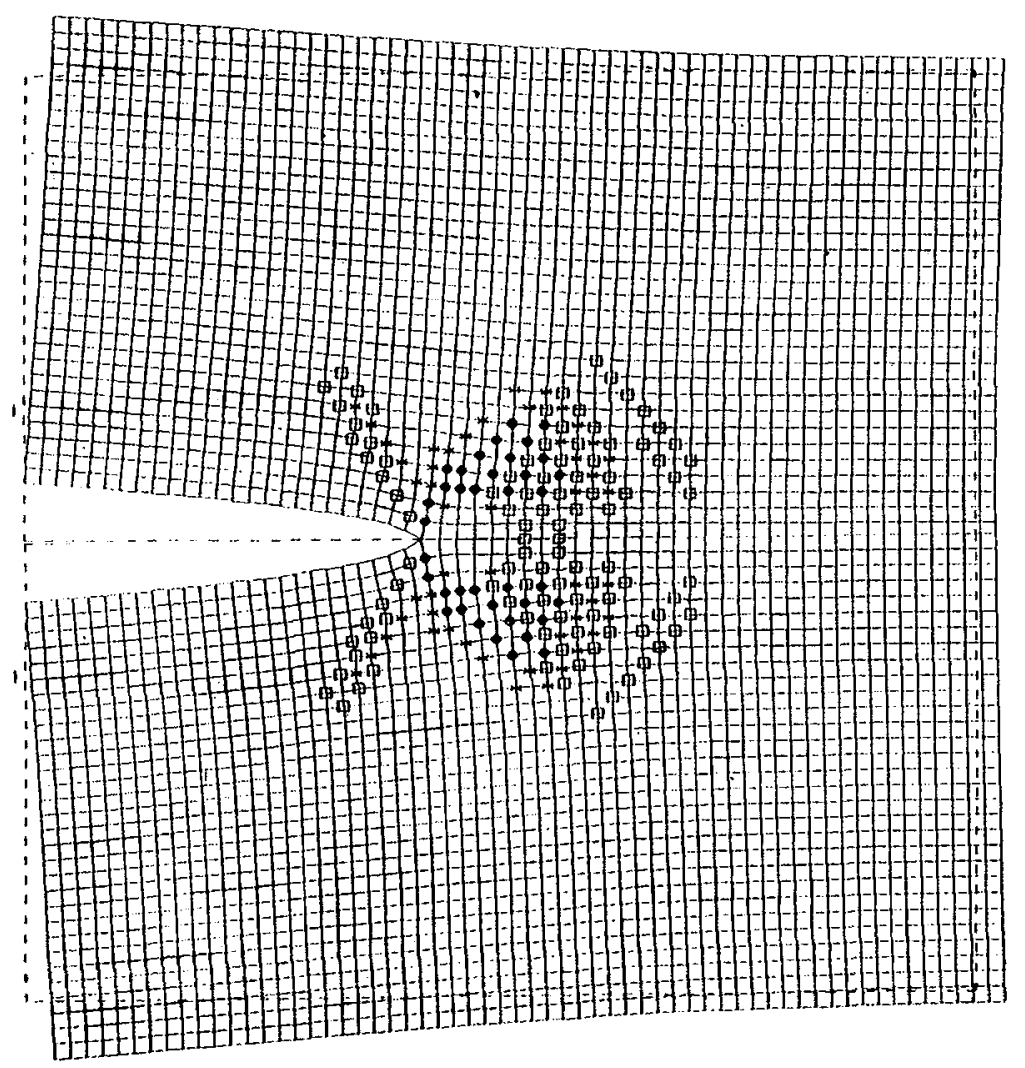

Fig. 7 - Computer simulation of stress induced martensite pattern near a crack under Mode I loading in an ordered B2 NiAl alloy. The martensite phase is an ordered face centered tetragonal phase with the ratio of long to short tetragonal dimension (c/a) about 1.3. Open squares indicate transformation zones where the long tetragonal axis is parallel to the applied stress and normal to the crack plane. Solid squares are zones with the long axis parallel to the crack plane. Spacing between finite element nodes is ten unit cell distances (about $30 \AA$ ).

\section{CONCLUSIONS}

1. Controlling the distribution of nuclei for an impending phase transformation can be very important for controlling the microstructure and ultimate properties of a material.

2. Much remains to be learned about the nature of nucleation centers in a wide variety of phase transformations.

3. The discovery of new metastable transformations often requires knowing how to avoid the "normal" phase transformations. 


\section{ACKNOWLEDGEMENTS}

A heartfelt appreciation is offered to Dr. Georges Martin and his group at CEREM, Saclay for their very supportive hospitality while the author was on sabbatical leave, and also the deepest gratitude to the Fulbright Commission for a Senior Research Scholarship which provided the time and freedom to write this article. Jack Christian is thanked for my liberal use of his excellent treatment of classical nucleation theory along with his graphic devices. John Cahn has also helped very much in my understanding of nucleation concepts over the years. Any mistakes in this article are however solely mine. Much appreciation goes also to the Dept. of Energy, Division of Materials Research which has been supporting the transformation toughening simulation studies.

\section{BIBLIOGRAPHY}

${ }^{1}$ I. S. Servi and D. Turnbull - Acta Metall. 14, 161 (1966)

${ }^{2}$ J. W. Cahn, Acta Metall. 4, 49 (1956); Ibid. 5, 168 (1957)

${ }^{3}$ The Theory of Transformations in Metals and Alloys, J. W. Christian, Pergamon Press (1975)

${ }^{4}$ P. J. Clemm and J. C. Fisher, Acta Metall. 3, 70 (1955)

5 P. C. Clapp, Mat. Sci. and Eng., A127, 189-195 (1990)

6 D. J. Green, R. H. J. Hannink and M. V. Swain: Transformation Toughening of Ceramics, CRC Press, Inc., Florida (1989)

7 P. C. Clapp, C. S. Becquart, Y. Shao, Y. Zhao and J. A. Rifkin - to be published

${ }^{8}$ Y. Zhao, Ph. D. thesis, University of Connecticut (1993) 